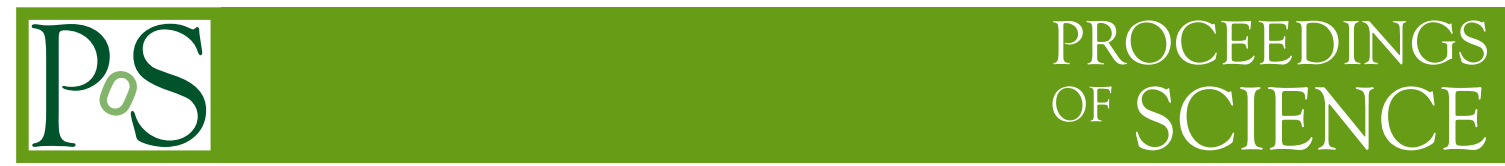

\title{
Radiative and semileptonic decays in Chiral Perturbation Theory
}

\section{Johan Bijnens*}

Department of Theoretical Physics, Lund University,

Sölvegatan 14A, SE 22362 Lund, Sweden

E-mail: bijnens@thep.lu.se

I give a short overview of what has been done in radiative and semileptonic Kaon decays in Chiral Perturbation Theory. This includes for semileptonic decays the work which has been done to order $p^{6}$ including preliminary results of isospin breaking to that order in $K_{\ell 3}$. For the radiative decays I mainly present some results from recent work concerning $K_{\ell 3 \gamma}$.

Kaon International Conference

May 21-25, 2007

Laboratori Nazionali di Frascati dell'INFN

* Speaker. 


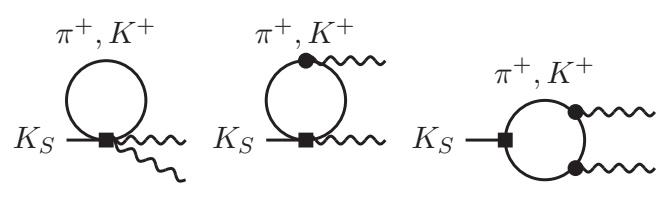

Figure 1: The order $p^{4}$ diagrams for $K_{S} \rightarrow \gamma \gamma$.

\section{Introduction}

In this talk I give a short overview of the work done on semileptonic and radiative semileptonic Kaon decays within the context of Chiral Perturbation Theory (ChPT). There is of course a lot more work done within ChPT relevant for Kaon decays than I can possibly do justice to within this short talk. Other theory talks with a very large ChPT content presented at this conference are those of V. Cirigliano, J. Prades, J. Gasser, G. Colangelo and C. Smith.

The Daphne report on semileptonic Kaon decays [1] still provides a very good introduction to the ChPT of semileptonic and radiative semileptonic Kaon decays. Below I will mainly comment on what has happened since then.

\section{Nonleptonic radiative decays}

Let me first remind you of one of the major successes of ChPT in radiative decays, the prediction $[2,3]$ from the diagrams in Fig. 1 of the decay $K_{S} \rightarrow \gamma \gamma$. The prediction for a branching ratio of $2.1 \cdot 10^{-6}$ has since been amply confirmed by experiment. There is some disagreement between the NA48 and KLOE results as discussed in the talk by M. Martini. However, both results are in reasonable agreement with the ChPT prediction. There are some estimates of the effect of final state interactions but no full order $p^{6}$ calculation exists.

More experimental results on different nonleptonic radiative decays can be found in the talks by E. Imbergamo, E. Cheu and M. Martini. These also discuss comparisons with ChPT.

\section{Semileptonic decays}

Most of these decays were studied in current algebra already. Gasser and Leutwyler then worked out $K \rightarrow \ell v$ (really $F_{K}$ ) and $K \rightarrow \pi \ell v$ in their seminal work on ChPT[4, 5]. The decay $K \rightarrow \pi \pi \ell v$ was worked out to order $p^{4}$ in $[6,7]$ and a dispersive estimate of the higher orders was done by [8]. The latter reference also evaluated the remaining form-factor $R$ to order $p^{4}$. Except for the $R$ formfactor, these calculations have all been performed to order $p^{6}$ now and will be discussed in more detail below. The decay $K_{e 5}$ is also kinematically possible. It has been evaluated to lowest order in ChPT by S. Blaser [9]. The resulting branching ratios vary from a few times $10^{-12}$ to a few times $10^{-11}$ and these processes are thus not very likely to be measured in the foreseeable future.

\section{1 $K_{\ell 2}$}

This decay, under the guise of the calculation of $F_{K}$ and $F_{\pi}$ for the corresponding $\pi_{\ell 2}$ decays, was performed to order $p^{6}$ in [10]. Its main use is the fitting of the low-energy constant (LEC) $L_{5}^{r}$ in ChPT. A typical convergence from [11], fit 10, is

$$
F_{K} / F_{\pi}=1.22=1.000+0.162+0.058, \quad F_{\pi} / F_{0}=1.000+0.135-0.075 .
$$


The value 1.22 is used here as input for $F_{K} / F_{\pi}$.

\section{$3.2 K_{\ell 3}$}

This decay is one the major sources of knowledge about the parameter $V_{u s}$. This was discussed in great detail at this conference. The theory overview was given by V. Cirigliano and a new Flavianet average was presented by M. Palutan. The measurements themselves were also discussed by several other speakers.

The corrections to $f_{+}(0)$ at order $p^{4}$ were first done in [12] and to all formfactors in [5]. The radiative corrections have been discussed at this conference by V. Cirigliano and can be found in [13]. Let me discuss here the calculation in the isospin limit to order $p^{6}$ [14] first, some results can also be found in [15]. First it was pointed out that $f_{+}(0)$ is in fact rather dependent on the curvature in the formfactor $f_{+}(t)$, as discussed in the experimental talks here, that curvature has since been measured, thus removing that uncertainty in the experimental determination of $f_{+}(0)$ in principle. Unfortunately, the experiments are not in full agreement due to the large correlations between the linear slope and the curvature. However, when using the pole form for $f_{+}(t)$ all experiments are in good agreement and agree reasonably well with the ChPT prediction of the curvature[14].

One of the problems with ChPT is the large number of parameters that appear at higher orders. However, for $f_{+}(0)=f_{0}(0)$ the needed order $p^{6}$ LECs, $C_{12}^{r}$ and $C_{34}^{r}$, are in principle determinable from the scalar formfactor slope and curvature:

$$
\begin{aligned}
f_{0}(t)= & 1-\frac{8}{F_{\pi}^{4}}\left(C_{12}^{r}+C_{34}^{r}\right)\left(m_{K}^{2}-m_{\pi}^{2}\right)^{2}+8 \frac{t}{F_{\pi}^{4}}\left(2 C_{12}^{r}+C_{34}^{r}\right)\left(m_{K}^{2}+m_{\pi}^{2}\right) \\
& +\frac{t}{m_{K}^{2}-m_{\pi}^{2}}\left(F_{K} / F_{\pi}-1\right)-\frac{8}{F_{\pi}^{4}} t^{2} C_{12}^{r}+\bar{\Delta}(t)+\Delta(0) .
\end{aligned}
$$

$\bar{\Delta}(t)$ and $\Delta(0)$ contain $n o C_{i}^{r}$ and only depend on the $L_{i}^{r}$ at order $p^{6}$. All needed parameters can thus in principle be determined experimentally. In practice this will be difficult, but dispersion theory can help producing relations between the slope and curvature, see e.g. the talk by E. Passemar at this conference. Thus measuring the scalar formfactor as precisely as possible should still help in improving the accuracy for $V_{u s}$. The final conclusion of [14] for the remainder was

$$
\Delta(0)=-0.0080 \pm 0.0057[\text { loops }] \pm 0.0028\left[L_{i}^{r}\right]
$$

where there is a sizable cancellation between the order $p^{4}$ and the pure loop order $p^{6}$ contribution.

As discussed in the experimental review on $K_{\ell 3}$ by M. Palutan, there is a small discrepancy between the determinations of $V_{u s}$ from $K_{\ell 3}^{+}$and $K_{\ell 3}^{0}$ decays. The ratio between these decays is well predicted to order $p^{4}$ and $e^{2} p^{2}$ in ChPT. There is thus a question whether the remainder is due to isospin breaking at higher order in ChPT. Such a calculation is underway [16] and preliminary results are shown in Tab. 1. There also the results of the isospin conserving calculation are shown for $f_{+}(0)$. What can be seen is that the order $p^{6}$ correction in general lowers the isospin breaking seen in the ratio. However, when the full order $p^{6}$ fit including isospin breaking [11] is taken into account there is an increase by about $0.5 \%$ compared to the old order $p^{4}$ results of $[5,12]$. The change in $m_{u} / m_{d}$ comes about half from the order $p^{6}$ corrections and half from the inclusion of corrections to Dashen's theorem. Dealing with $\pi^{0}-\eta$ mixing is somewhat more involved at order $p^{6}$ but can be dealt with[11, 16]. Whether all necessary order $p^{6}$ LECs can be determined similar to the isospin conserving case is under investigation[16]. 


\begin{tabular}{ccccccc}
\hline Decay & $p^{2}$ & $p^{4}$ & pure 2-loop & $L_{i}^{r}$ at $p^{6}$ & $C_{i}^{r}$ & total \\
\hline \multicolumn{7}{c}{ Iso conserving calculation } \\
$K^{0}$ & 1 & -0.02266 & 0.01130 & 0.00332 & $? ? ?$ & 0.99196 \\
$K^{+}$ & 1 & -0.02276 & 0.01104 & 0.00320 & $? ? ?$ & 0.99154 \\
\hline \multicolumn{7}{c}{$m_{u} / m_{d}=0.45$} \\
$K^{0}$ & 1 & -0.02310 & 0.01131 & 0.00325 & $? ? ?$ & 0.99146 \\
$K^{+}$ & 1.02465 & -0.01741 & 0.00379 & 0.00648 & $? ? ?$ & 1.01751 \\
ratio & 1.02465 & 1.0311 & & & & 1.0262 \\
\hline \multicolumn{7}{c}{$m_{u} / m_{d}=0.58$} \\
$K^{0}$ & 1 & -0.02299 & 0.01124 & 0.00325 & $? ? ?$ & 0.99150 \\
$K^{+}$ & 1.01702 & -0.01897 & 0.00657 & 0.00551 & $? ? ?$ & 1.01013 \\
ratio & 1.0170 & 1.0215 & & & & 1.0188
\end{tabular}

Table 1: Preliminary results of isospin breaking to order $p^{6}$ for $f_{+}(0)$ in $K_{\ell 3}[16] . m_{u} / m_{d}=0.58$ corresponds to the values used in $[12,5]$ while $m_{u} / m_{d}=0.45$ is the result of the full order $p^{6}$ fit[11].

\section{$3.3 K_{\ell 4}$}

This decay plays a major role in the discussion of $\pi \pi$ scattering. This was discussed at this conference by G. Colangelo, B. Bloch-Devaux and L. Di Lella, here I concentrate on the values of the formfactors. The lowest order result, $F=G=m_{K} /\left(\sqrt{2} F_{\pi}\right)$, was worked out by Weinberg. The order $p^{4}$ calculations were done around $1990[6,7]$ and a dispersive estimate of the higher orders appeared somewhat later [8]. The latter reference also evaluated the remaining form-factor $R$ to order $p^{4}$. The absolute values of $F$ and $G$ provide the best input for $L_{i}^{r}, i=1,2,3$. The full order $p^{6}$ calculation was done in [17, 18] for $F$ and $G$ and in [19] for the vector formfactor $H$. It was found that the full calculation gave a higher order correction somewhat larger than the dispersive estimate but that the ChPT series was generally converging. An updated fit of the ChPT LECs to the new NA48 $K_{\ell 4}$ measurements, including the information from $\pi \pi$ scattering, is certainly needed.

\section{Radiative semileptonic Kaon decays}

\section{1 $K_{\ell 2 \gamma}$}

The internal Bremsstrahlung contribution to this decay was known for a long time. The order $p^{4}$ contribution was done in [20] and the order $p^{6}$ in [21] for the axial formfactor. The vector formfactor is predicted at order $p^{4}$ by the anomaly and is known to order $p^{6}[19]$. The corrections are of the expected size for both formfactors.

\section{2 $K_{\ell 3 \gamma}$}

The inner Bremsstrahlung contribution was calculated using current algebra methods[22]. The order $p^{4}$ calculation was performed in [20] and a thorough analysis of the higher order corrections, using the fact that cuts are far away from the physical region, can be found in the papers [23, 24, 25]. 


\begin{tabular}{ccccccc}
\hline$E_{\gamma}^{\text {cut }}$ & $\theta_{e \gamma}^{\text {cut }}$ & $R^{\mathrm{IB}} \cdot 10^{2}$ & $R \cdot 10^{2}$ & $c_{1} \cdot 10^{3}$ & $c_{2} \cdot 10^{4}$ & $c_{3} \cdot 10^{4}$ \\
\hline $30 \mathrm{MeV}$ & $20^{\circ}$ & 0.640 & $0.633 \pm 0.002$ & $12.5 \pm 0.4$ & $-5.4 \pm 0.3$ & $16.9 \pm 0.4$ \\
$30 \mathrm{MeV}$ & $10^{\circ}$ & 0.925 & $0.918 \pm 0.002$ & $11.1 \pm 0.3$ & $-4.7 \pm 0.2$ & $15.0 \pm 0.3$ \\
$10 \mathrm{MeV}$ & $20^{\circ}$ & 1.211 & $1.204 \pm 0.002$ & $7.5 \pm 0.2$ & $-3.2 \pm 0.2$ & $10.1 \pm 0.2$ \\
$10 \mathrm{MeV}$ & $10^{\circ}$ & 1.792 & $1.785 \pm 0.002$ & $6.7 \pm 0.2$ & $-2.8 \pm 0.1$ & $9.0 \pm 0.1$ \\
$10 \mathrm{MeV}$ & $26^{\circ}-53^{\circ}$ & 0.554 & $0.553 \pm 0.001$ & $5.7 \pm 0.1$ & $-2.4 \pm 0.1$ & $7.5 \pm 0.1$ \\
\hline
\end{tabular}

Table 2: The ratios $R$ in $K_{\ell 3 \gamma}$ decay as a function of the $K_{\ell 3}$ formfactor parameters[23].

It was found there that in the ratios

$$
R\left(E_{\gamma}^{\mathrm{cut}}, \theta_{e \gamma}^{\mathrm{cut}}\right)=\Gamma\left(K_{e 3 \gamma}^{ \pm}, E_{\gamma}^{*}>E_{\gamma}^{\mathrm{cut}}, \theta_{e \gamma}^{*}>\theta_{e \gamma}^{\mathrm{cut}}\right) / \Gamma\left(K_{e 3}^{ \pm}\right)
$$

many of the uncertainties drop out. Studies of how the structure functions can be determined experimentally can also be found there. As an example I show the table for the ratios as a function of the $K_{\ell 3}$ slopes[23].

\section{Conclusions}

Chiral Perturbation Theory has been extremely useful in semileptonic and radiative Kaon decays. I have given a very fast overview of existing calculations and presented some preliminary results for the order $p^{6}$ isospin breaking in $K_{\ell 3}[16]$. Let me finish by pointing out that in addition to all the results discussed above, many of these decays provide good tests of the anomaly, including its sign $[1,19,26]$. Much of what I discussed here has been reviewed to order $p^{4}$ in [1] and to order $p^{6}$ in [27].

\section{Acknowledgments}

I like to thank the organizers for a very enjoyable meeting and in getting together a great program in Kaon physics. This work is supported in part by the European Commission RTN network, Contract MRTN-CT-2006-035482 (FLAVIAnet), the European Community-Research Infrastructure Activity Contract RII3-CT-2004-506078 (HadronPhysics) and the Swedish Research Council.

\section{References}

[1] J. Bijnens, G. Colangelo, G. Ecker and J. Gasser, Semileptonic Kaon Decays, arXiv:hep-ph/9411311.

[2] J. L. Goity, The decays $K_{S}^{0} \rightarrow \gamma \gamma$ and $K_{L}^{0} \rightarrow \gamma \gamma$ in the chiral approach, Z. Phys. C 34 (1987) 341.

[3] G. D’Ambrosio and D. Espriu, Rare Decay Modes of the K Mesons in the Chiral Lagrangian, Phys. Lett. B 175 (1986) 237.

[4] J. Gasser and H. Leutwyler, Chiral Perturbation Theory: Expansions In The Mass Of The Strange Quark, Nucl. Phys. B 250 (1985) 465. 
[5] J. Gasser and H. Leutwyler, Low-Energy Expansion Of Meson Form-Factors, Nucl. Phys. B 250 (1985) 517.

[6] C. Riggenbach, J. Gasser, J. F. Donoghue and B. R. Holstein, Chiral Symmetry And The Large $N_{C}$ Limit In $K_{\ell 4}$ Decays, Phys. Rev. D 43 (1991) 127.

[7] J. Bijnens, $K_{\ell 4}$ decays and the low-energy expansion, Nucl. Phys. B 337 (1990) 635.

[8] J. Bijnens, G. Colangelo and J. Gasser, $K_{\ell 4}$ decays beyond one loop, Nucl. Phys. B 427 (1994) 427 [arXiv:hep-ph/9403390].

[9] S. Blaser, $K_{e 5}$ decays in ChPT, Phys. Lett. B345 (1995) 287 [arXiv:hep-ph/9410368].

[10] G. Amorós, J. Bijnens and P. Talavera, Two-point functions at two loops in three flavour ChPT, Nucl. Phys. B568 (2000) 319 [arXiv:hep-ph/9907264].

[11] G. Amorós, J. Bijnens and P. Talavera, QCD isospin breaking in meson masses, decay constants and quark mass ratios, Nucl. Phys. B 602 (2001) 87 [arXiv:hep-ph/0101127].

[12] H. Leutwyler and M. Roos, Determination Of The Elements $V_{u s}$ and $V_{u d}$ of the Kobayashi-Maskawa Matrix, Z. Phys. C 25 (1984) 91.

[13] V. Cirigliano, M. Knecht, H. Neufeld, H. Rupertsberger and P. Talavera, Radiative corrections to $K_{\ell 3}$ decays, Eur. Phys. J. C 23 (2002) 121 [arXiv:hep-ph/0110153].

[14] J. Bijnens and P. Talavera, $K_{\ell 3}$ decays in ChPT, Nucl. Phys. B 669(2003)341 [arXiv:hep-ph/0303103].

[15] P. Post and K. Schilcher, $K_{\ell 3}$ form factors at order $p^{6}$ in ChPT, Eur. Phys. J. C 25 (2002) 427 [arXiv:hep-ph/0112352].

[16] J. Bijnens and K. Ghorbani, work in progress.

[17] G. Amorós, J. Bijnens and P. Talavera, Phys. Lett. B 480 (2000) 71 [arXiv:hep-ph/9912398].

[18] G. Amorós, J. Bijnens and P. Talavera, Nucl. Phys. B 585 (2000) 293 [Erratum-ibid. B 598 (2001) 665] [arXiv:hep-ph/0003258].

[19] L. Ametller, J. Bijnens, A. Bramon and F. Cornet, Semileptonic $\pi$ and $K$ decays and the chiral anomaly at one loop, Phys. Lett. B 303 (1993) 140 [arXiv:hep-ph/9302219].

[20] J. Bijnens, G. Ecker and J. Gasser, Radiative semileptonic kaon decays, Nucl. Phys. B 396 (1993) 81 [arXiv:hep-ph/9209261].

[21] C. Q. Geng, I. L. Ho and T. H. Wu, Axial-vector form factors for $K_{\ell 2 \gamma}$ and $\pi_{\ell 2 \gamma}$ at order $p^{6}$ in ChPT, Nucl. Phys. B 684 (2004) 281 [arXiv:hep-ph/0306165].

[22] H. W. Fearing, E. Fischbach and J. Smith, Current algebra, $\bar{K}_{\ell 3}^{0}$ form-factors, and radiative $\bar{K}_{\ell 3}^{0}$ decay, Phys. Rev. D 2 (1970) 542.

[23] B. Kubis, E. H. Müller, J. Gasser and M. Schmid, Aspects of radiative $K_{e 3}^{+}$decays, Eur. Phys. J. C 50 (2007) 557 [arXiv:hep-ph/0611366].

[24] J. Gasser, B. Kubis, N. Paver and M. Verbeni, Radiative $K_{e 3}$ decays revisited, Eur. Phys. J. C 40 (2005) 205 [arXiv:hep-ph/0412130].

[25] E. H. Müller, B. Kubis and U. G. Meissner, T-odd correlations in radiative $K_{\ell 3}^{+}$decays and ChPT, Eur. Phys. J. C 48 (2006) 427 [arXiv:hep-ph/0607151].

[26] J. Bijnens, ChPT and anomalous processes, Int. J. Mod. Phys. A 8 (1993) 3045.

[27] J. Bijnens, ChPT beyond one loop, Prog. Part. Nucl. Phys. 58 (2007) 521 [arXiv:hep-ph/0604043]. 